\title{
Biodegradable Quantum Composites for Synergistic Photothermal Therapy and Copper- enhanced Chemotherapy
}

Han-Xiao Tang ${ }^{a, b}$, Chen-Guang Liu ${ }^{a, b}$, Jian-Ting Zhang ${ }^{a, b}$, Xiang Zheng ${ }^{a, b}$, Da-Yun Yang ${ }^{c}$, Ranjith Kumar Kankala ${ }^{a, b}$, Shi-Bin Wang ${ }^{a, b}$, and Ai-Zheng Chen ${ }^{a, b, *}$

${ }^{a}$ Institute of Biomaterials and Tissue Engineering, Huaqiao University, Xiamen 361021, P.R. China

${ }^{\mathrm{b}}$ Fujian Provincial Key Laboratory of Biochemical Technology (Huaqiao University), Xiamen 361021, P. R. China

c Institute for Translational Medicine, School of Basic Medical Sciences, Fujian Medical University, Fuzhou 350108, P. R. China

${ }^{*}$ Corresponding author: Email: azchen@hqu.edu.cn (A. -Z.C)

Tel./Fax: +86 5926162326 


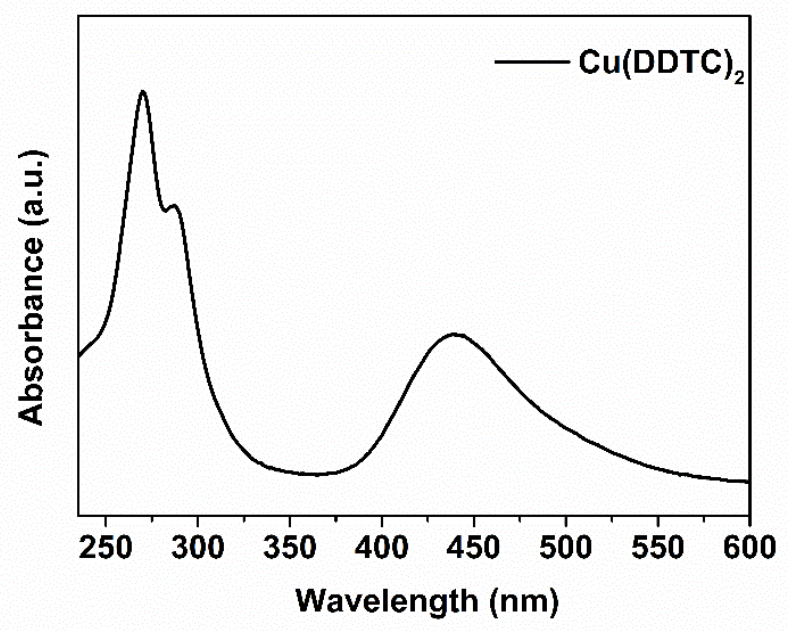

Figure S1. UV-vis spectrum of $\mathrm{Cu}(\mathrm{DDTC})_{2}$
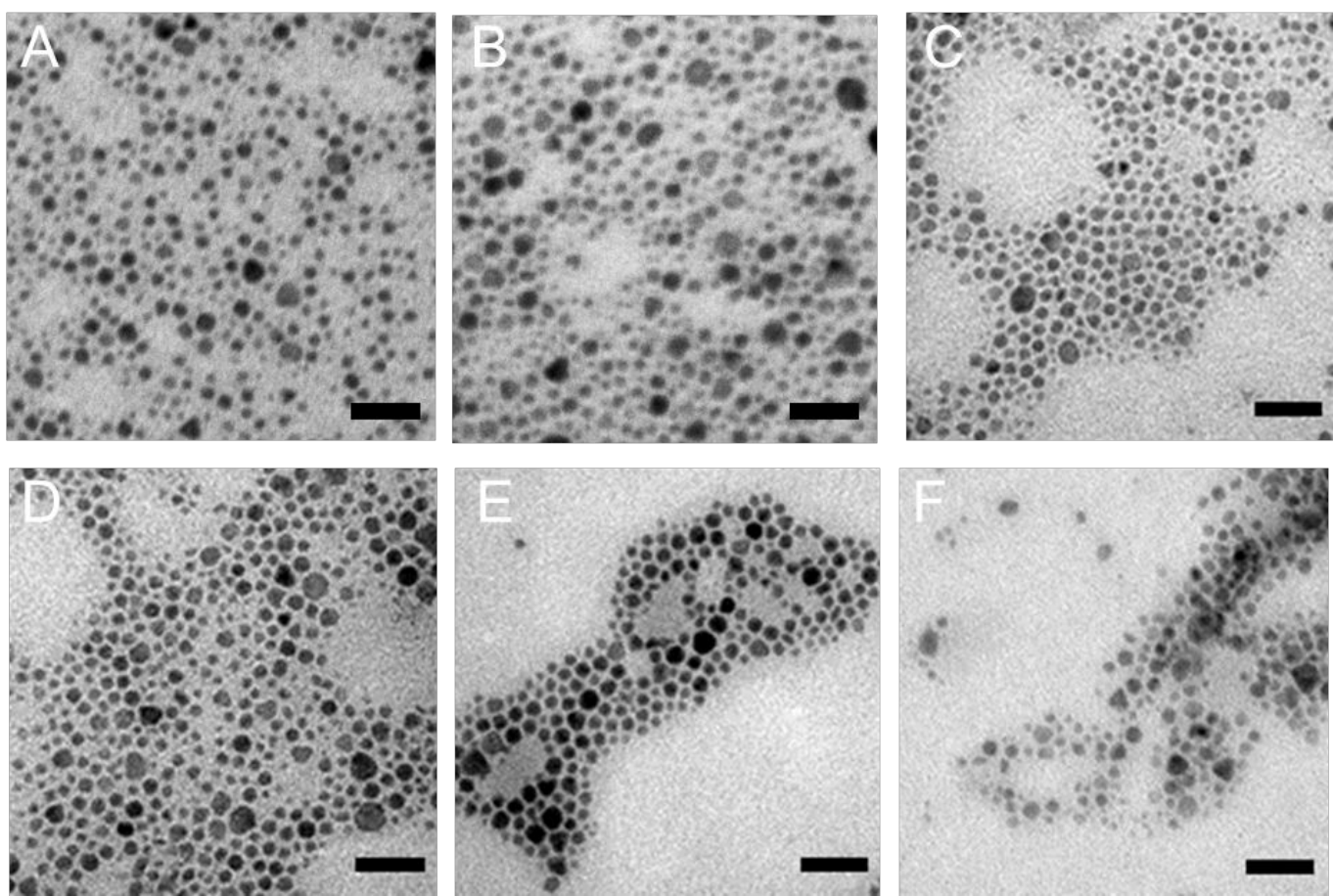

Figure S2. TEM images of CuS-DDTC NDs prepared at different mass ratios. A. 50:1. B. 25:1. C. 10:1. D. 1:1. E. 0.5:1. F. 0.25:1. Scale bar-50 nm. 
Cus

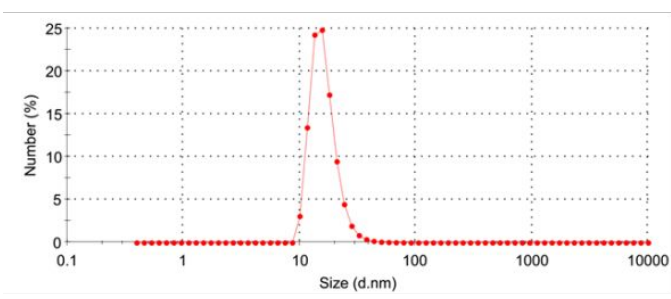

50:1

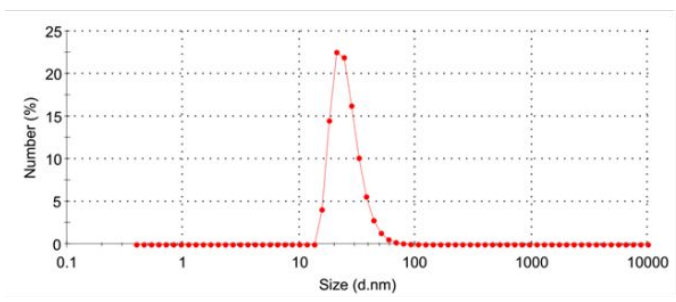

10:1

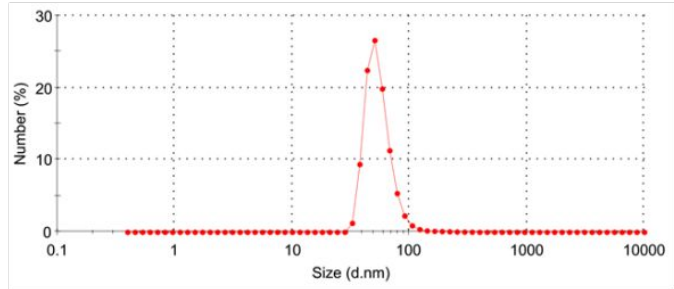

0.5:1

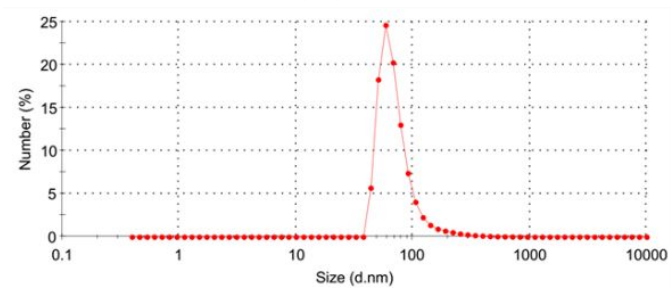

100:1

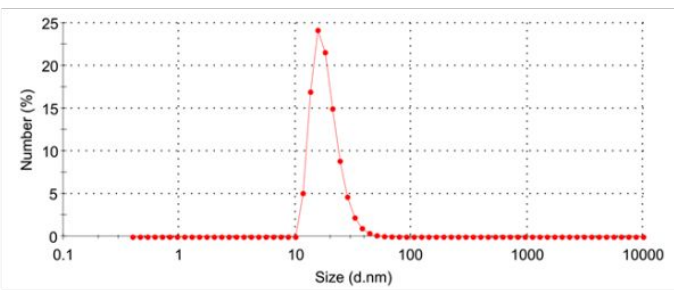

25:1

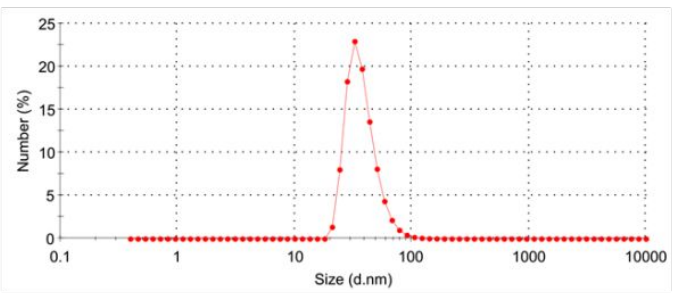

1:1

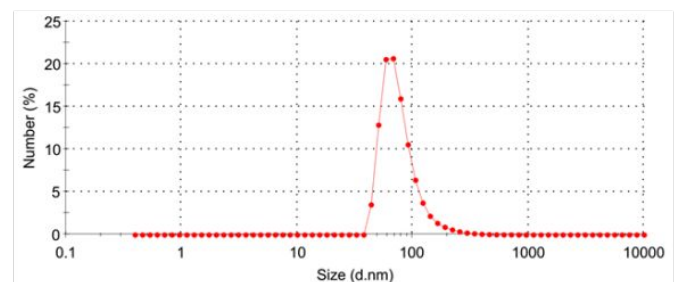

$0.25: 1$

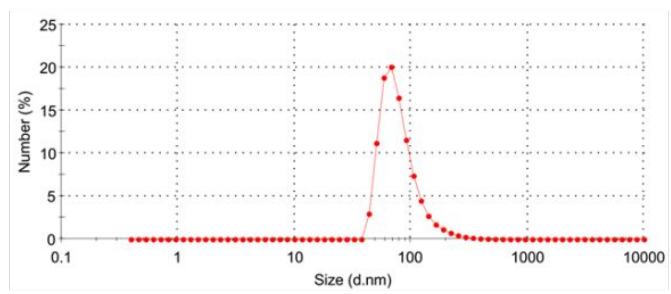

Figure S3. DLS of CuS NDs and CuS-DDTC NDs at different mass ratios in the aqueous solution. 

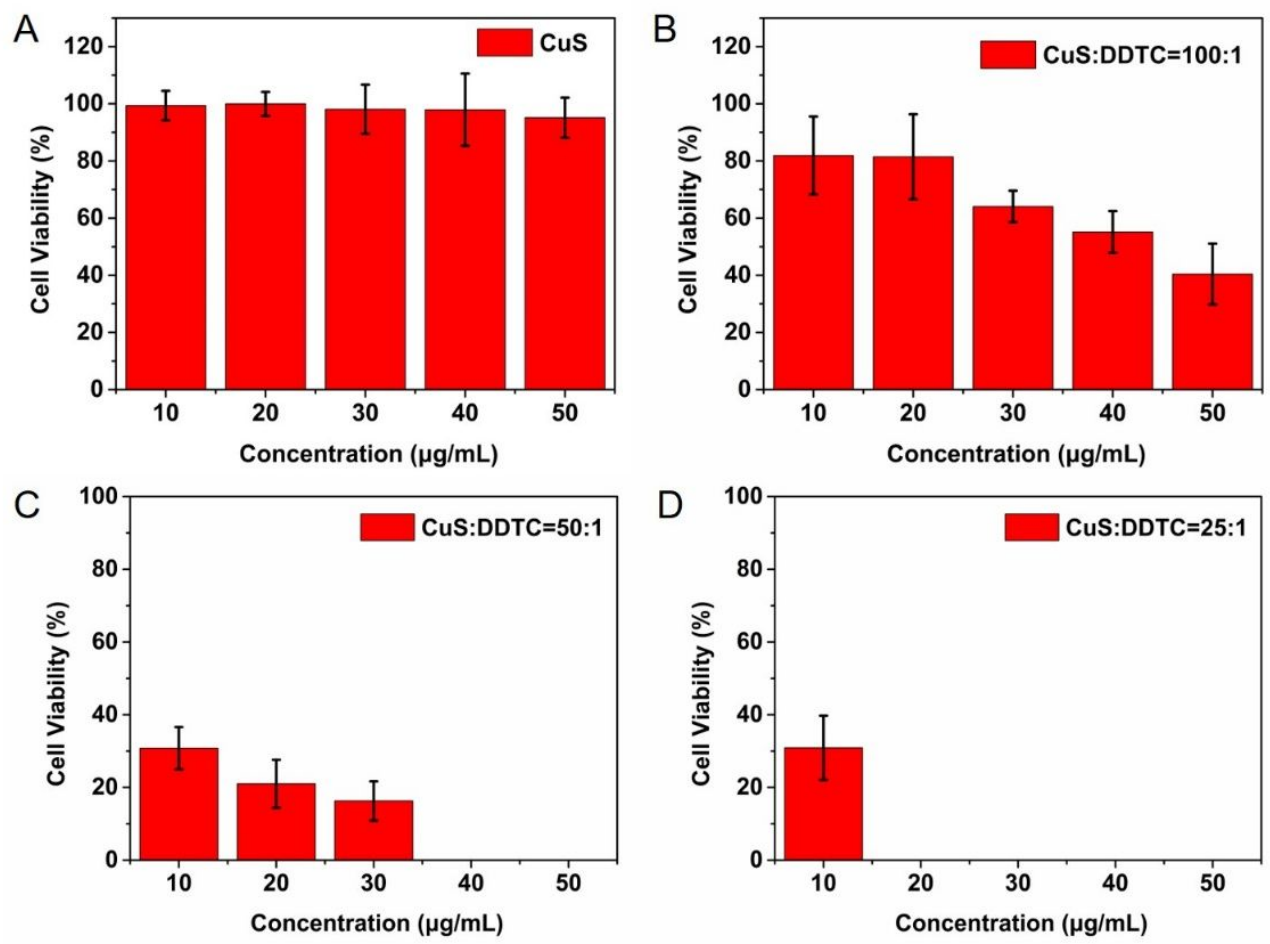

Figure S4. Viabilities of the HUVECs after incubation with (A) CuS NDs and CuS-DDTC NDs prepared at different mass ratios (B) 100:1, (C) 50:1, and (D) 25:1.
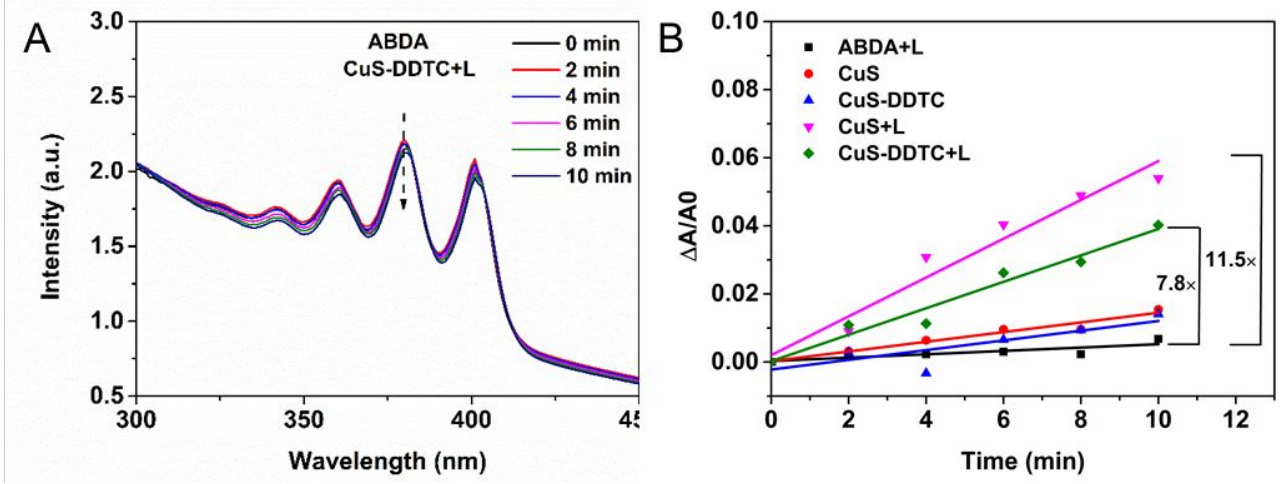

Figure S5. A. Change of UV-Vis absorption spectra of ABDA. B. degradation reaction rates of ABDA calculated from the absorbance of the ABDA at $380 \mathrm{~nm}$ under different treatments. 


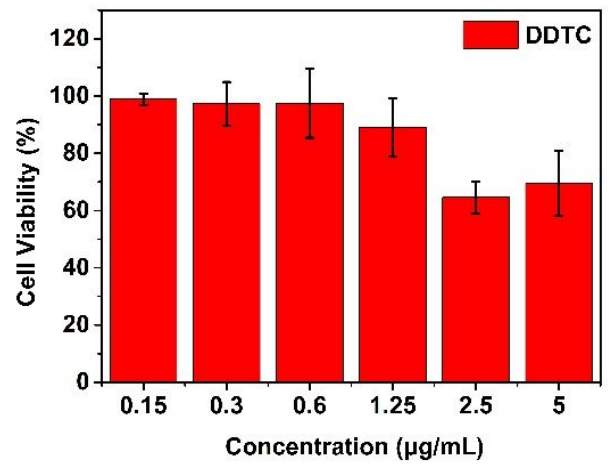

Figure S6. Cell viabilities of 4T1 cells after incubation with DDTC for $24 \mathrm{~h}$.

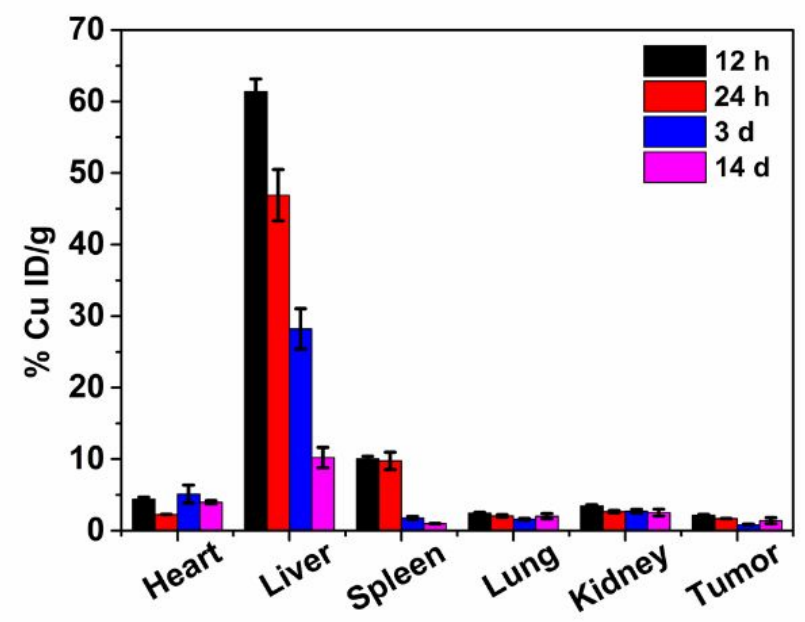

Figure S7. Distribution of CuS NDs in the tumor and major organs at different time points. 

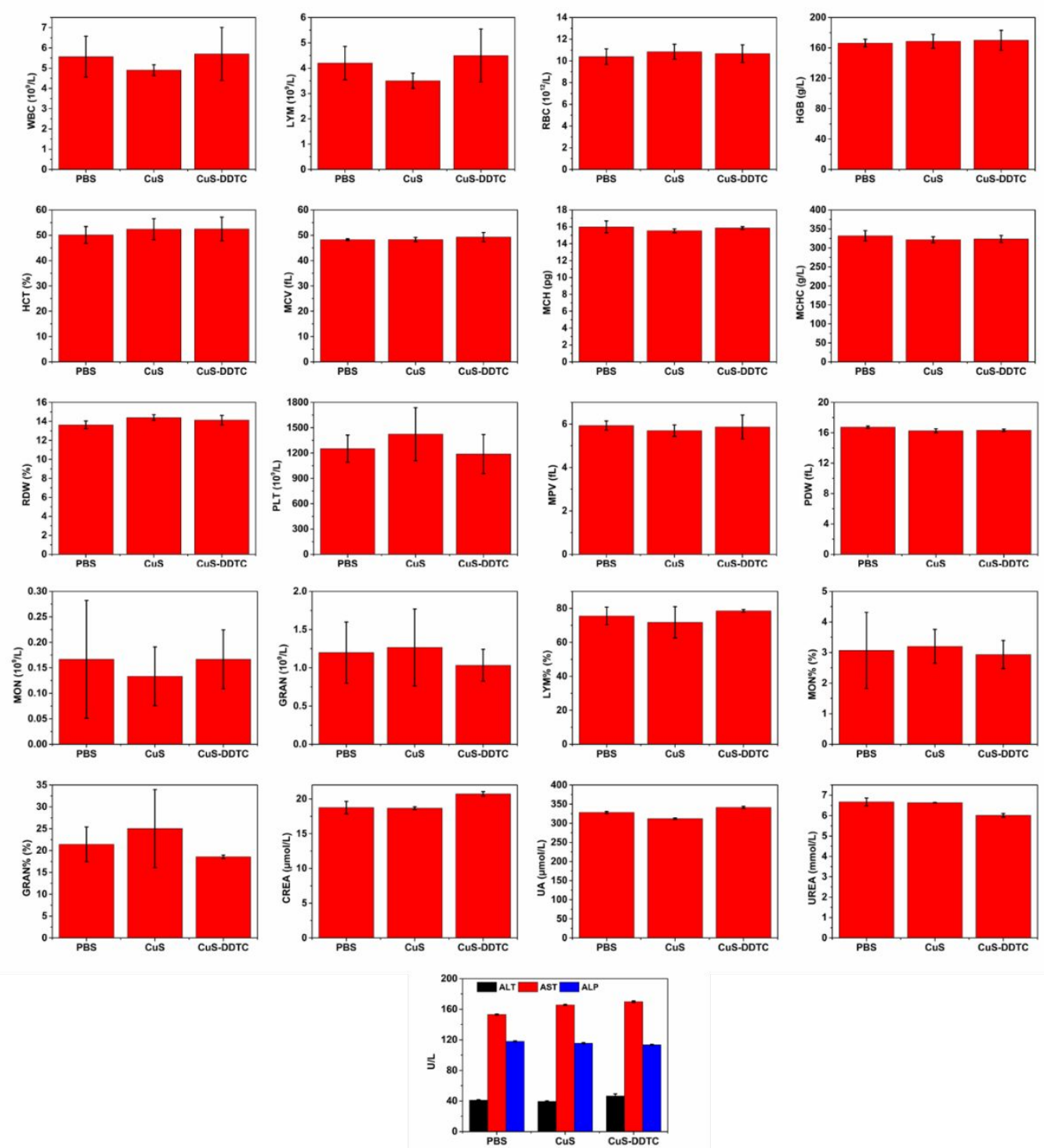

Figure S8. Hematological assessment. The related indices of KM mice after 14d intravenously injected with PBS, CuS NDs, and CuS-DDTC NDs. 


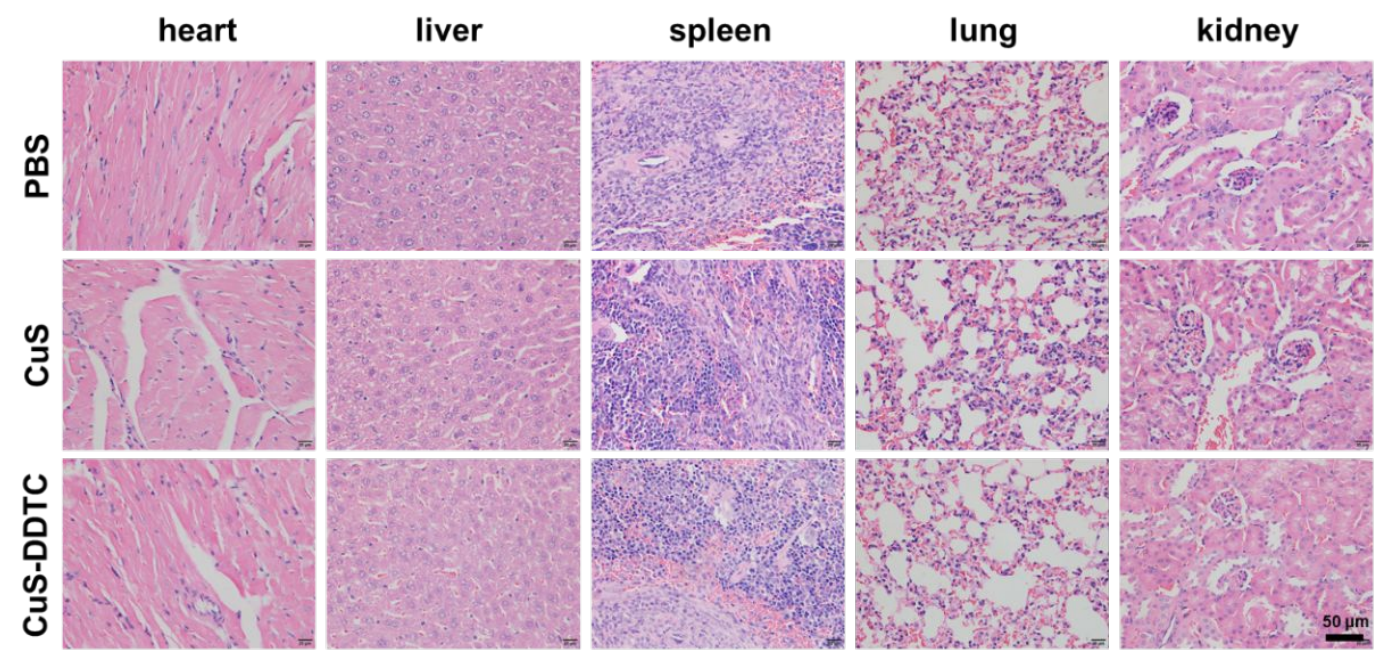

Figure S9. H\&E stained images of the major organs of the mice after $14 \mathrm{~d}$ intravenously injected with PBS, CuS NDs, and CuS-DDTC NDs.
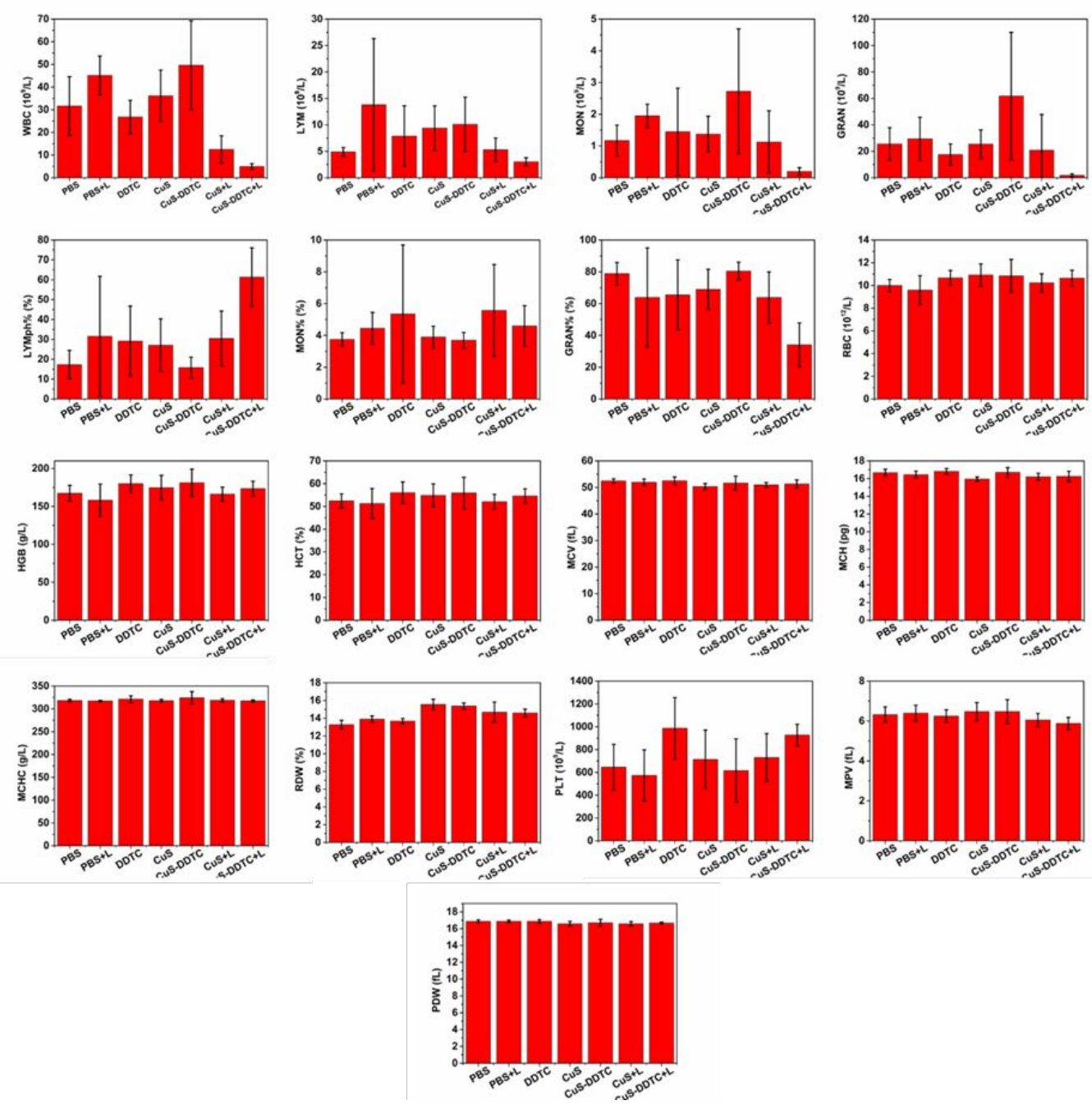

Figure S10. Hematological assay. The related indices of 4T1-TBM after $14 \mathrm{~d}$ with different treatments. 